\title{
Molar characterization and analytical UPLC method development of matrix impurity, disregards impurity, specified impurity associated undetectable impurity of laboratory drug Isatin
}

\author{
Rahul Hajare* \\ Department of Health Research, Ministry of Health and Family Welfare, New Delhi, India
}

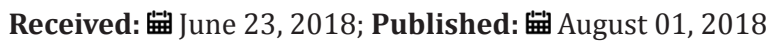

*Corresponding author: Rahul Hajare, Department of Health Research, Ministry of Health and Family Welfare, New Delhi, India

\begin{abstract}
This is out line work on the process development for the impurity detection of Isatin from synthetic occurring and several new methods have been developed during the pursuit of this research. The article traces the evolution of various approaches and provides a comparison for overall efficiency. UPLC method has been developed and validated for simultaneous estimation of matrix impurity, disregards impurity; specified impurity associated undetectable impurity in pure synthetic formulations. Separation was carried out using column Hypersil ODS C18 (250 $\mathrm{mm}$ x 4.6mm x $5 \mu \mathrm{m}$ particle size) in isocratic mode using mobile phase composition pH 6.0 ammonium acetate Buffer: Acetonitrile (68:32) v/v and UV detection at $310 \mathrm{~nm}$. The impurities were eluted at a flow rate of $1.0 \mathrm{~mL} / \mathrm{min}$. The average retention times for matrix impurity, disregards impurity, specified impurity associated undetectable impurity were 2.86 and $3.67 \mathrm{~min}, 4.54 \mathrm{~min}$ respectively. The method was validated according to the ICH guidelines.
\end{abstract}

Keywords: Matrix Impurity; Disregards Impurity; Specified Impurity Associated Undetectable Impurity; UPLC; Ich Guidelines; Process Development

\section{Introduction}

Purity or limits of impurity and its coping measurements for drug products present a challenge to pharmacopeial standards-setting of a drug product over time is at issue, the same analytical methods that are stability-indicating are also purity-indicating. Resolution of the active ingredient(s) from preparation presents the same qualitative problem [1]. Thus, many monographs for Pharmacopeial preparations feature chromatographic assays. Where more significant impurities are known, some new monographs set forth specific limit tests. In general, however, this pharmacopeia does not repeat impurity tests in subsequent preparations where those appear in the monographs of drug substances and where those impurities are not expected to increase. Here close monitoring of unique recombination formation of impurity amplified and sequenced [2,3]. The implementation of the new monograph requirement concerning matrix impurity, disregards impurity and specified impurity associated undetectable impurity in synthetic

drug substances aim at better quality characterization of those human products and thus at better medicinal product in the market. The invention discloses drug development and methods and it has updated regularly.

Matrix impurity: Not more than 1.5 times the area of the principal peaks in the chromatogram obtained with refence solution (c) (0.3 per cent).

3.2. Disregards impurity: Not more than the area of the principal peaks in the chromatogram obtained with refence solution (c) (0.2 per cent).

\subsection{Specified impurity associated undetectable} impurity: Not more than the area of the principal peaks in the chromatogram obtained with refence solution (c) ( 0.10 per cent). Total impurity not more than 0.6 per cent the area of the principal peaks in the chromatogram obtained with refence solution(c). 
a) Dissolve $25.0 \mathrm{mg}$ of reference compound in the mobile phase and dilute to $50.0 \mathrm{ml}$ with the mobile phase.

b) Dilute $1.0 \mathrm{ml}$ of the test solution to $50.0 \mathrm{ml}$ with the mobile phase. Dilute $1.0 \mathrm{ml}$ this solution to $10.0 \mathrm{ml}$ with the mobile phase.

Mobile phase: Mix 13 volumes of acetonitrile and 83 volumes of a $2.45 \mathrm{gm} / \mathrm{L}$ solution of phosphoric acid previously adjusted to pH 3.0 with triethylamine.

Flow rate: $1.5 \mathrm{ml} / \mathrm{min}$.

Detection: Spectrophotometer at $278 \mathrm{~nm}$.

Injection: $50 \mathrm{ul}$ of the test solution and refence solution (c)

Test solution: Dissolve $25.0 \mathrm{mg}$ of the substance to be examined in the mobile phase and dilute to $50.0 \mathrm{ml}$ with the mobile phase.

Correction factor: For the calculation of content multiply the peaks areas of the following impurities by the corresponding correction factors.

\section{Matrix impurity: 6.7.}

\section{Disregards impurity: 0.7 .} 0.6 .

\section{Specified impurity associated undatable impurity:}

\section{Assay Liquid chromatography (2.2.29 European} Pharmacopiea 5.0) as described in the test for related substances with the following modifications

\section{Injection}

$10 \mu \mathrm{l}$; inject the test solution and reference solution (a). Impurities with product in above section monograph components are under general notices and requirements as well as general ordinary impurities. Addressed topic of purity or impurity has come up into focus when handling validation of compendial procedure of laboratory product $1 \mathrm{H}$ - Indole 2,3, Dione (Isatin).

\section{Monograph of Pure Synthetic Isatin}
a. Chemical Name: 1H-indole-2,3- dione.
b. Synonym: 2,3- Indolinedione.
c. Molecular Formula: C8H5NO2.
d. Molecular Weight: $147.1308 \mathrm{~g} / \mathrm{mol}$.
e. Appearance: Orange red solid.
f. Melting point: 2000C (392 F, 473K).
g. Solubility: Soluble in water.

h. $\mathrm{pH}: 6$ and 9 at temperature up to $500 \mathrm{C}$.

i. Conditions to avoid: Over Heat.

j. Other adverse effects: Negligible ecotoxicity.

k. Heave metals: Maximum 20 ppm.

l. Sulphated ash: maximum 0.1 per cent, determined on $1.0 \mathrm{~g}$ in platinum crucial.

m. Purity (by UPLC): 99\%.

Assay: Liquid chromatography as describe in the test for related substance with the following modification.

a. Injection: $5 \mu$ l inject the test solution and reference solution (a).

b. Calculate the percentage content of C8H5NO2.

c. Moisture Content: NMT 0.5\% wt./wt.

d. Usage: A key raw material used in the manufacturing of various Anti-bacterial and Antiviral Agent.

e. Storage: Store in a cool place protected from light.

f. Hazards Note: Irritant.

g. Hazard statements: Causes skin irritation, causes serious eye irritation, may cause respiratory irritation.

h. Protection: If on skin: wash with plenty of water.

i. Exposure hazards: In combustion emits toxic fumes.

j. Workplace exposure limits: Close monitoring required.

k. Water: Maximum 1percent, determined on $0.200 \mathrm{~g}$.

\section{Limits}

Correction factors: for the calculation of contents, multiply the peak areas of the following impurities by the corresponding correction factor: impurity inherent $=0.7$; impurity ignored $=0.6$; use the chromatogram obtained with reference solution (b) and the type chromatogram supplied with the Isatin to identify the corresponding peaks.

\section{Inherent Impurity}

0.25 times the area of the principal peak in the chromatogram obtained with reference solution (c) (0.05 per cent). Ignore impurity, not more than the area of the principal peak in the chromatogram obtained with reference solution (c) (0.2 per cent).

a. Dissolve $25.0 \mathrm{mg}$ of isatin in the mobile phase and dilute to $50.0 \mathrm{ml}$ with mobile phase.

b. Dilute $1.0 \mathrm{ml}$ of the test solution to $50.0 \mathrm{ml}$ with the mobile phase. Dilute $1.0 \mathrm{ml}$ of this solution to $10.0 \mathrm{ml}$ with the mobile phase. 


\section{Mobile phase}

The buffer solution for the UPLC method was prepared by dissolving $0.5 \mathrm{~g}$ of sodium carbonate and $0.5 \mathrm{~g}$ sodium bicarbonate in $1000 \mathrm{~mL}$ of Milli-Q grade water ( $\mathrm{pH}$ 9.71). The content of the mixture was sonicated prior to the analysis. The mobile phase consisted of buffer and acetonitrile in equal ratio.

\section{Disposal}

Disposal of balance sample shall carry out as per disposal SOP.

\section{Acknowledgement}

This study has been guided under the supervision and guidance of Renowned Immunologist Respected Dr Ramesh S.
Paranjape, Long-Time NARI Director and Renowned Scientist, India. This method has established under international regulatory requirements. This research has done at R \& D Dept. as officer at Aarti Drugs Ltd. Trapur MIDC Mumbai.

\section{References}

1. Rahul Hajare, Smita Kulkarni, Madhuri Thakar, Ramesh Paranjape (2016) Technology development and design of novel 1, 3, 5-tri substituted-1hindole-2, 3-dione hiv-1 inhibitors with displays strategic nanomolar cytotoxicity 5(6): 391-397.

2. Keitel S (2006) Impurity Profiles in Active Pharmaceutical Ingredients. EU / Swiss medic GMP Workshop Beijing University.

3. Josephs JL, Sanders M, Shipkova P (2007) Detection and Characterization of Pharmaceutical Metabolites, Degradants and Impurities by the Application of MS / MS Software Algorithms; Technical Program.

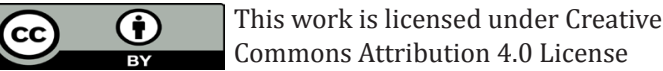

To Submit Your Article Click Here:

Submit Article

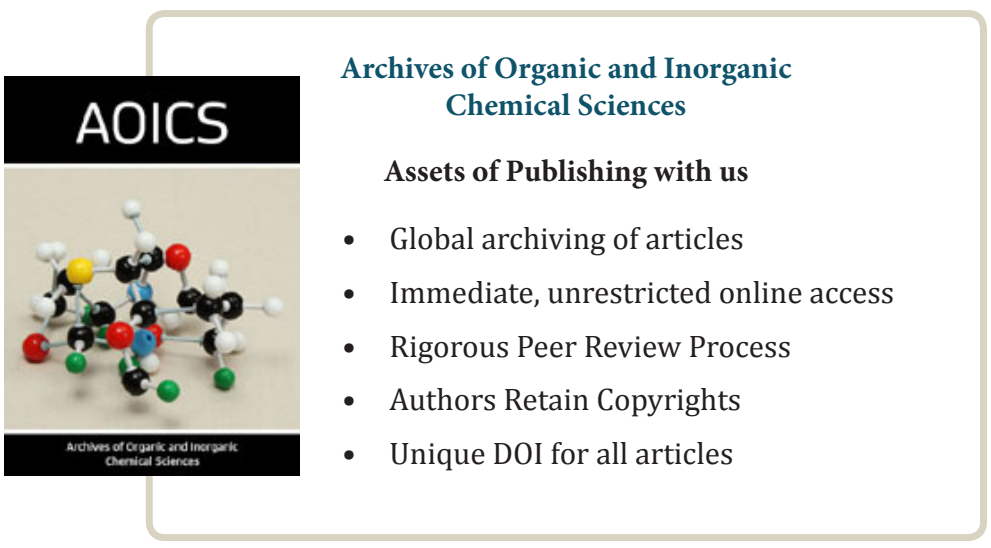

